\title{
Cáncer y salutogénesis: una revisión bibliográfica
}

\section{Cancer and salutogenesis: A bibliographic review}

\author{
Sergio I. Escamilla Sánchez ${ }^{1}$ \\ Citación: Escamilla S., S.I. (2021). Cáncer y salutogénesis: una revisión bibliográfica. Psicología \\ y Salud, 31(1), 131-141. https://doi.org/10.25009/pys.v31i1.2683.
}

\section{RESUMEN}

\begin{abstract}
Al ser el cáncer una de las principales causas de preocupación de los sistemas de salud de muchos países se vuelve necesario hacer una revisión del estado actual de la literatura que aborde el tema desde el punto de vista de la salud y no de la enfermedad, que es el objeto del constructo salutogénesis. Si bien se considera una relación polémica el vínculo entre el cáncer y los pensamientos saludables, muy contraria a la existente con la depresión o ansiedad, y por tanto la bibliografía es escasa, en este trabajo se han tomado en cuenta los resultados de una búsqueda de las publicaciones aparecidas entre los años 2009 y 2019, principalmente. La importancia de la intervención psicológica que promueve en los pacientes un estado mental y emocional en el que predominen pensamientos y sentimientos saludables se hace evidente en cuanto se logra la disminución de los síntomas y la remisión acelerada de la condición al construirse y reforzarse un círculo virtuoso al aumentar el bienestar y la calidad de vida.
\end{abstract}

Palabras clave: Bienestar; Calidad de vida; Cáncer; Salutogénesis.

\begin{abstract}
As cancer is one of the main concerns of health systems worldwide, it becomes necessary to review the current state of the research literature addressing this area from the perspective of health and not disease, which is the object of the salutogenesis construct. There is a controversial relationship between cancer and healthy thoughts and, unlike those that relate them to depression or anxiety, research studies are scarce. The results of a search and review for publications between 2009 and 2019 seem to point toward a documentable association. The importance of psychological interventions that promote a mental and emotional state where healthy thoughts and feelings predominate seems better supported since they achieve the reduction of symptoms and the accelerated remission of the condition. These thoughts and feelings probably reinforce a virtuous circle leading to increased well-being and quality of life in cancer patients.
\end{abstract}

Keywords: Cancer; Quality of life; Salutogenesis; Wellbeing.

\section{Modelo salutogénico}

$\mathrm{E}$ n 1946 se elaboró la Constitución de la Organización Mundial de la Salud (OMS), en la que los estados participantes declararon los principios básicos para la felicidad, las relaciones armoniosas y la seguridad de los pueblos. El primero de dichos principios define que la salud es un estado de completo bienestar físico, mental y social, y no solamente la ausencia de afecciones o enfermedades (OMS, 1946). Se reconoce también la inclusión de un paradigma que pone el foco en la salud y no en la enfermedad.

\footnotetext{
${ }^{1}$ Universidad de Baja California, Calle Av. Juan F. Parkinson 160, Fracc. Parque Ecológico, 63173 Tepic, Nay., México, tel. (311)133-42-42, correos electrónicos: sies8@msn.com y ubcposgrados@hotmail.com. Artículo recibido el 8 de julio de 2019 y aceptado el 15 de junio de 2020.
} 
Entre las condiciones para conservar el estado de salud tiene una importancia particular la psicológica. Con base en estudios hechos a sobrevivientes del holocausto nazi, en los años 70, el norteamericano de raíces hebreas, Aaron Antonovsky comenzó a desarrollar el llamado modelo salutogénico, el cual influyó considerablemente en el pensamiento de los médicos y científicos del comportamiento por la explicación dada de los factores psicológicos que forman las bases de la salud humana. Antonovsky (1979) utilizó las siguientes tres preguntas claves para la fundación de su teoría: “¿Por qué las personas se mantienen saludables a pesar de estar expuestas a tantas influencias perjudiciales? ¿cómo se las arreglan para recuperarse de enfermedades? y ¿qué tienen de especial las personas que no enferman a pesar de la tensión más extrema?" (cf. Rivera, Ramos, Moreno y Hernán, 2011).

El autor también parte del supuesto de que hay que hacer énfasis en el origen de la salud y el bienestar, enfocando la atención en su mantenimiento y progreso. Antonovsky rechaza la hipótesis de que los factores estresantes son intrínsecamente negativos y se muestra a favor de que puedan tener consecuencias saludables o benéficas dependiendo de sus características y de la capacidad de las personas para resolverlos. Antonovsky (1987) desarrolló su teoría de la salud, a la que denominó salutogénesis, con base en estudios llevados a cabo -como se ha dicho antes- con quienes sufrieron los horrores de la época nazi, muchos de los cuales no solo se recuperaron y sobrevivieron sino que prosperaron, concluyendo así que existen factores psicológicos que causan la salud. A partir de tales resultados abrió una gran rama de investigación que dio por resultado el concepto de sentido de coherencia ( $\mathrm{SOC}$, por las siglas en inglés de Sense Of Coherence, en lo sucesivo) (Eriksson, 2007).

El autorreconocimiento, su equivalente en inglés self-concept clarity (SCC), hace referencia a un aspecto estructural por medio del cual las creencias propias del individuo están definidas con claridad y confianza, consistentes internamente y estables. Campbell et al. (1996) señalan que una SCC baja se asocia de forma independiente con alta neurosis, baja autoestima, bajo grado de conciencia, baja aceptabilidad, autoanálisis crónico, conciencia baja del estado interno y una forma melancólica de atención centrada en uno mismo. Adicionalmente, Dias de Andrade (2013) propone que la evaluación del SCC -sea alta o baja en términos de la claridad y certidumbre que tiene el sujeto respecto a sí mismo- tiene una fuerte correlación positiva con su velocidad para procesar y recuperar información.

Desde la perspectiva de la psicología profunda, la psicología de arquetipos de Jung, la subjetividad del bienestar o el malestar está plenamente justificada con su concepto de la imaginación activa, ya que todo depende de cómo vemos las cosas y no cómo son por ellas mismas. La menor de las cosas con significado en la vida es siempre más valiosa que la mayor de las cosas sin significado (Jung, 1997). Todas las obras del hombre tienen su origen en la imaginación activa; por ello, dice Jung, la actividad creativa de la imaginación libera al hombre de sus ataduras a la resignación de lo que le sucede y le asciende a la posición del que hace que suceda. El hombre es completamente humano solo cuando está participando activamente.

Este último concepto junguiano, el de que lo que importa es cómo se reacciona a los sucesos cotidianos, que no tienen cualidad moral por sí mismos más que el valor intrínseco de los sucesos mismos, es un concepto que confronta los modelos científicos materialistas, que buscan la objetividad más absoluta posible del objeto estudiado.

Con la aparición del psicoanálisis y el estudio de la lingüística hay un auge de las hipótesis que sostienen que la palabra tiene un notorio grado de influencia sobre el cuerpo. Por ejemplo, Alemán y Larriera (2005) sostienen que a partir del desarrollo del psicoanálisis freudiano el discurso es una mentira verídica, esto es, que la verdad no es una exacta representación del relato al suceso, sino que la verdad se produce en el decir a otro $y$ en el efecto que sus palabras operan sobre el que habla. Por ello, dichos autores postulan que hay inconsciente allí donde se habla y que este inconsciente se da como aquello que "sabe" más de lo que el hablante quiere decir. Presentan el origen del inconsciente desde el punto de vista del psicoanálisis del modo siguiente: "El psicoanálisis no tiene como tarea dilucidar hasta dónde llega lo psíquico y hasta dónde lo biológico, sino qué tipo de operaciones se producen y cuáles son las 
consecuencias de este hecho constitutivo del ser hablante: la afectación del cuerpo por la palabra. Esta relación, de por sí indecidible e incalculable, entre la palabra y el cuerpo deja en los hablantes un sedimento de naturaleza simbólica al que Freud caracterizó como Inconsciente" (Alemán y Larriera, 2005).

En las teorías psicoanalíticas y lingüísticas hay una inclinación a darle importancia al síntoma, a la patología, porque se estudia la forma en que el estado de no-salud se expresa a través de las expresiones del inconsciente. En el lacanismo toma fuerza la noción de que el síntoma se hace patente en el modo en que el cuerpo y la palabra están implicados; la palabra afecta al cuerpo: lo desvitaliza, lo fragmenta y lo modifica. Más aún, en la psicología profunda se llega a extremos como los de Hillman (1975), quien declara que la psique tiene una habilidad autónoma para crear enfermedad, morbidez, desorden, anormalidad y sufrimiento en cualquier aspecto del comportamiento y a experimentar una imagen de vida a través de esa perspectiva deforme y afligida.

Es evidente entonces que la indisoluble relación entre psique y cuerpo es tal que la acción de una produce cambios en el otro. Por ello, es importante estudiar cómo dichos cambios pueden ocurrir tanto hacia la patología como hacia la salud, en ese continuo que hay entre ambos. De acuerdo con Rodríguez, Casas, Contreras y Ruano (2015), el narcisismo, desde el más patológico hasta el más saludable, pasa hoy día por la senda obligada del cuerpo, controlándolo y asimismo sometiéndolo. Es así que es posible ver que actualmente se da gran importancia a modificar y mantener el cuerpo por medio de factores externos, como la dieta, el ejercicio y los fármacos, pero además hay que fomentar el reconocimiento de que la psique también colabora en tales modificaciones de manera tan o más importante.

Si el foco de atención se pone en la salud y no en la enfermedad, procurando que el estado saludable prevalezca sobre el patológico, el beneficio para los individuos y para la sociedad en su conjunto será considerable en muchas áreas; particularmente, en el área de las políticas de salud pública, el beneficio económico será tangible. Ruano y Mercé (2014) subrayan esta última idea afirmada por Lindström y Eriksson (2006) al señalar que con buena salud mental y buena calidad de vida, gracias a un buen nivel de SOC, las personas enfermarían en menos ocasiones y habría menos incapacidades o prejubilaciones por enfermedad, y de esta manera se obtendrían beneficios económicos.

\section{Psicooncología: salutogénesis vs depresión}

La psicooncología es la rama de la psicología que se ocupa de evaluar, investigar e intervenir en los comportamientos de los individuos afectados por el cáncer en todas sus fases, desde el diagnóstico, tratamiento, postratamiento, seguimiento de los que superan la condición o última etapa de la vida, en su caso. De acuerdo con Cabrera, López, López y Arredondo (2017), la psicooncología se encarga de las situaciones de vida y de las características culturales, psicosociales y espirituales que influyen en la aparición y tratamiento del cáncer, y su repercusión en los pacientes y en su entorno familiar y social, cuyo objetivo es contribuir al desarrollo y la evaluación de terapias psicológicas efectivas que permitan reducir la morbilidad psicológica y mejorar la calidad de vida de los pacientes.

Por su parte, Mota, Aldana, Bohórquez, Martínez y Peralta (2018) enlistan diferentes formas de apoyo psicológico para pacientes con cáncer particularmente de mama-, entre las que destacan las siguientes: terapias cognitivo-conductuales, intervención psicoeducativa, psicoterapia breve, psicoterapia de grupo, terapias alternativas y complementarias, e intervención psicológica al final de la vida. En la descripción que se hace de ellas, se destaca que las terapias cognitivo-conductuales son útiles para eliminar pensamientos depresivos, autocondenatorios, de culpa, desesperanza o de autocompasión, mediante la modificación de la forma de razonamiento. La intervención psicoeducativa está enfocada en la rehabilitación física y la modificación de la dieta. La psicoterapia breve ayuda a disminuir el malestar psicosocial y contribuye a que el paciente recobre la funcionalidad y el control de su vida; mientras que las de grupo son útiles en el cambio de actitudes, conductas y emociones por medio de la reflexión conjunta, el intercambio de experiencias, el autoconocimiento y el apoyo mutuo. Las terapias alternativas y complementarias se utilizan para mejorar el bienestar 
físico o psicológico y la capacidad del cuerpo para combatir el cáncer, aplicándose en situaciones de ansiedad, miedo y dolor. La intervención que se utiliza al final de la vida se realiza con el propósito de lograr la aceptación y la tranquilidad para poder cerrar los aspectos que generan desazón en la etapa final.

Ante los estados de la falta de salud, la perspectiva de la salutogénesis subraya el cuidadoso uso del pensamiento y el lenguaje, privilegiando la utilización de conceptos con connotación positiva sobre sobre aquellos con negativa. De acuerdo con Antonovsky (1987), el argumento principal del constructo de salutogénesis sostiene que para el individuo un fuerte sentido de coherencia (SOC) es un predictor de resultados positivos para su salud, a la vez que tiene una alta correlación con la forma en que ese individuo le da sentido a su mundo y usa los recursos requeridos para responder a las situaciones, percibiendo si sus respuestas son significativas y tienen sentido emocionalmente.

En la formulación del SOC, Abernethy (2011) define tres componentes: Comprensibilidad: la creencia de que las cosas suceden de manera ordenada y predecible, sintiendo que se entienden los eventos y que se pueden predecir razonablemente los que sucederán en el futuro; Manejabilidad: la creencia de que se tienen las aptitudes, las habilidades, el apoyo, la ayuda o los recursos necesarios para atender las situaciones, y que estas son manejables y están bajo control, y Significancia: la creencia de que los sucesos en la vida son interesantes y una fuente de satisfacción, que valen la pena y que hay una buena razón o propósito para atenderlos.

De manera inversa, un sentido de coherencia débil y una actitud más negativa hacia el cáncer, mirando a las víctimas con pena, considerando a la enfermedad como una sentencia de muerte o abrigando el miedo a esta, predicen un alto nivel de estrés y malestar psicológico. Gerasimcik-Pulko, Pileckaite-Markoviene, Bulotiene y Ostapenko(2009) estudiaron a mujeres lituanas diagnosticadas con cáncer de mama, encontrando que las pacientes con un SOC más alto disfrutaban de una mejor calidad de vida durante las etapas tempranas de su condición, a diferencia de las que tenían un SOC más bajo. Las primeras raramente se quejaron de síntomas atribuibles a los efectos secundarios de los tratamientos, porque pudieron afrontar mejor el estrés relacionado con el diagnóstico. Aunque en el estudio se reconoce que hay otras investigaciones que hallan que el nivel de SOC no es una disposición estable, o que en pacientes sometidas a quimioterapia no hay necesariamente una correlación con su calidad de vida, las conclusiones de la investigación específica muestran que pacientes con cáncer de mama con un mayor sentido de coherencia experimentan menos efectos secundarios del tratamiento y tienen una mejor calidad de vida relacionada con la salud en el periodo postoperatorio temprano.

Volanen, Lahelma, Silventoinen y Suominen (2004) señalan que la intención de Antonovsky al introducir el concepto de SOC era la de proporcionar una alternativa al abordaje de los factores adversos para así preguntarse qué factores son los que más probablemente pueden proteger a las personas de enfermar, ya que aquellas con un SOC fuerte evalúan situaciones potencialmente peligrosas o incontrolables como desafíos y, por lo tanto, pueden mantenerse saludables ante ellas.

Los recursos generalizados de resistencia (GRR, por sus siglas en inglés de Generalized Resistance Resource) contribuyen al SOC y se definen como las condiciones de vida y la educación que comienzan en la infancia, y que a partir de la juventud y la adultez se manifiestan en la vida laboral, la familiar y la social. En el estudio de los citados autores en una muestra finlandesa se midieron el SOC y los GRR, obteniendo como resultado que los niveles de ambos constructos son similares en hombres y mujeres, además de que "el SOC parece reflejar, en particular, el bienestar o malestar psicoemocional de hombres y mujeres, y se asocia con sus relaciones cercanas, de pareja y amigos, así como con el apoyo social, las condiciones de trabajo, las situación de empleo y las condiciones de vida en la niñez" (Volanen et al., 2004).

Alivia, Guadagni y Roberti (2011) afirman que la enfermedad es un desafío al bienestar físico, psicológico y espiritual que trae repercusiones a la identidad y al contexto social; por ello, desarrollan el concepto de una medicina centrada en la persona, considerando a esta última como una entidad integral. Tal perspectiva exige una vi- 
sión que va más allá del mero enfoque patogénico, que diagnostica a través de pruebas en busca de signos de enfermedad y prescribe tratamientos en consecuencia para que el individuo sea declarado curado cuando nuevas pruebas ya no detecten el padecimiento y los parámetros se hayan normalizado. No obstante -señalan los autores-, la práctica diaria se caracteriza por personas que sufren de enfermedades crónicas, en las que, con frecuencia, existe una discrepancia entre sus resultados bioquímicos y la forma en que ellas se sienten. La salud, concluyen, es un concepto que va más allá de la ausencia de enfermedades y que incluye el bienestar físico, psicológico y espiritual, lo que está en consonancia con el constructo de salutogénesis, que nace de la exploración de las razones que se presentan en individuos que se mantienen saludables aun ante situaciones adversas y perniciosas, mientras que otras, encarando las mismas condiciones, caen enfermas.

La validación de instrumentos para medir el SOC se ha efectuado por un número considerable de investigadores en diversas partes del mundo. En la región hispanoamericana, Saravia, Iberico y Yearwood (2014) llevaron a cabo la validación de la Escala SOC-13 (13 por el número de reactivos que la componen) en una muestra de 448 individuos peruanos; utilizando el análisis estadístico apropiado, hallaron que la escala mostró propiedades psicométricas apropiadas respecto a la consistencia interna, la validez de criterios y la estructura de factores.

Alivia et al. (2011) proponen que cada intervención médica debería procurar reforzar el sentido de coherencia de la persona, así como su resistencia fisiológica, psicológica y espiritual. Los citados autores recomiendan que los profesionales de la salud debieran ser entrenados para proveer cuidados salutogénicos desde su formación profesional temprana, ya que el mismo aprendizaje de los conceptos fortalecen sus propios SOC y estado de salud. Al mismo tiempo, describen al SOC como un constructo que debe ser fortalecido, pues no es algo que posee o no el individuo de manera estática, sino que puede construirse y fortalecerse a lo largo del tiempo:

Hay un creciente cuerpo de investigación para todos los grupos de edades, diferentes antece- dentes socioeconómicos y culturales, que muestra cómo se relaciona un SOC fuerte con una mejor salud, envejecimiento más saludable y [como] un factor protector contra la adicción al alcohol. A la inversa, un soc débil está relacionado con una peor salud y un estado de ánimo más bajo. Aunque el soc se desarrolla naturalmente en los primeros 30 años de vida, no tiene una orientación estática. Puede ser fortalecido a través de la actividad personal y el cuidado (Alivia et al., 2011).

Agregando a la relación del sentido de coherencia el bienestar de los individuos, Wainwright et al. (2007) llevaron a cabo una investigación con una muestra de más de 18 mil personas en Norfolk (Reino Unido), con el propósito de probar la hipótesis de que las diferencias individuales en el SOC están asociadas a la elección de estilos de vida más saludables, independientemente de la clase social y del nivel de educación, hallando que aquellos individuos con un SOC más fuerte fumaban menos (28\%), eran menos inactivos físicamente $(36 \%)$ y consumían una mayor cantidad de frutas, vegetales y fibra, todo ello con independencia de su edad, género, clase social y educación. Por consiguiente, los autores proponen el fortalecimiento del SOC como una ayuda en el diseño de intervenciones para la promoción de la salud a futuro, y declaran que: "Individuos con un SOC fuerte pueden ser más capaces, por lo tanto, de adoptar un estilo de vida saludable y, por ejemplo, es más probable que respondan a consejos relacionados con la salud. Adicionalmente, los individuos con un SOC débil pueden participar en conductas menos saludables - por ejemplo, fumar cigarrillosporque son menos capaces de lidiar con el estrés diario" (Wainwright et al., 2007).

Gustavsson-Lilius, Julkunen, Keskivaara, Lipsanen, y Hietanen (2010) revisan una creciente literatura científica que reporta que los pacientes y sus parejas, cuidadores y familiares cercanos, están estrechamente ligados en el proceso de apoyo del enfermo, y que se influyen los unos a los otros, a lo cual se denomina "efecto transitivo" o "de cruce", sin importar el tipo o la etapa del cáncer. Se ha encontrado también que la cura del paciente se acelera y que el estrés de los cuidadores mejora más cuando se atienden las características de ambos, y no solo las del paciente individual- 
mente. Los citados autores emplean el constructo optimismo disposicional para referir las expectativas generalizadas de que sucederán cosas buenas, que en algunas investigaciones ha mostrado ser un amortiguador del estrés que producen las circunstancias desafiantes.

Acerca del optimismo y su efecto en la adaptación a las enfermedades hay trabajos específicos que prueban una alta correlación positiva. Por ejemplo, Friedman et al. (2005) llevaron a cabo un estudio entre pacientes de cáncer de mama de origen multiétnico para descartar los posibles sesgos que puede producir investigar poblaciones predominantemente caucásicas, encontrando que el optimismo, el someterse al tratamiento y el disponer de apoyo social explicaban en buena medida la varianza del bienestar funcional del individuo y su bienestar social y familiar, mientras que la historia familiar de cáncer de mama y el pesimismo se relacionaban con perturbaciones del humor, sin importar el origen étnico de las pacientes. Los autores concluyen que "el optimismo es un predictor de buena adaptación a una variedad de enfermedades somáticas, incluyendo diferentes tipos de cánceres".

En el otro extremo del optimismo se halla la depresión. Al respecto, Berenzon, Lara, Robles y Medina (2013) afirman que las personas que sufren depresión tienen mayores probabilidades de padecer enfermedades cardiovasculares y respiratorias, diabetes y cáncer, todas ellas condiciones crónicas y responsables de más de $60 \%$ de las muertes en el mundo. La relación entre el cáncer y la depresión se vuelve un círculo, pues quien padece cáncer puede desarrollar síntomas depresivos en algún momento de la enfermedad, y la depresión aumenta la probabilidad de desarrollar cáncer.

La depresión limita la capacidad de cuidar de sí mismo y disminuye la calidad de vida; adicionalmente, en los pacientes de cáncer aumenta la estancia hospitalaria y disminuye el cumplimiento de la terapia (Pousa, Miguelez, Hernández, González y Gaviria, 2015). Los mencionados autores agregan que «la depresión está infradiagnosticada en los pacientes oncológicos, quizá por la creencia de que "es normal que estén tristes"». Se identifica que solamente entre 10 y $33 \%$ de los pacientes con cáncer se deprime. Los instrumen- tos de diagnóstico más utilizados para evaluar tal condición son la Escala de Ansiedad y Depresión Hospitalaria (HADS), el Cuestionario de Salud General (GHQ-28) y el Screening Psicosocial para el Cáncer (PPS-CAN).

Es importante disponer de modalidades de tratamiento que mitiguen los efectos de la depresión. Tradicionalmente se emplean la psicofarmacología y la psicoterapia; sin embargo, Pousa et al. (2015) introducen la relevancia de las intervenciones de atención plena (mindfulness), relajación y uso de la imaginación, cuyas aplicaciones se han llevado a cabo en diversas instituciones, destacando sobre todo la terapia dialéctica conductual (DBT), la terapia de aceptación y compromiso (ACT), la terapia cognitiva basada en mindfulness (MBCT) y la reducción del estrés basada en la atención plena (MBSR). En conjunto, los resultados registrados reportan una reducción de los niveles de cortisol, la tasa cardíaca y las frecuencias cardíaca y respiratoria, así como la regulación de parámetros inmunológicos tales como el aumento de la producción de células NK y la reducción de citoquinas. En el área psicológica, se observa asimismo un impacto positivo tanto en los pacientes como en sus parejas o cuidadores. También se ha visto una reducción del efecto del estrés asociado al desencadenamiento y el miedo al cáncer, la activación del sistema inmunológico mediante la instauración de creencias positivas, el incremento de la motivación para el cambio del estilo de vida, el fortalecimiento del deseo de vivir, el enfrentamiento de la desesperanza y la modificación de las creencias del enfermo respecto a la enfermedad.

En un estudio efectuado con 140 pacientes en tratamiento, Guerrero, Prepo y Loyo (2016) concluyeron que "existe la necesidad de planificar intervenciones dirigidas a potenciar la autotrascendencia en pacientes con cáncer con la finalidad de mitigar los síntomas asociados a ambos trastornos"; respecto a la definición de autotrascendencia apuntan que «la mayoría de las definiciones que involucran la autotrascendencia están asociadas con un ser superior, donde la fe en un poder externo y el esfuerzo por el logro de un gran ideal son vistos como una expresión de la espiritualidad en los individuos. Esta interrelación conlleva una "necesidad espiritual" que permite a la persona mantener, aumentar o recuperar creencias, la fe o 
llevar a cabo obligaciones de tipo religiosas con la finalidad de suplir el vacío que hay en su interior»».

Los resultados obtenidos por Guerrero et al. (2016) muestran que la dimensión de preocupaciones sociales y estrés guarda una relación negativa con la autotrascendencia; es decir, a mayores niveles de esta, menor es la preocupación social y el estrés; Lo mismo ocurre con la depresión: a mayores niveles de autotrascendencia, menores niveles de depresión; sin embargo, entre ansiedad y autotrascendencia no se encontró una relación estadísticamente significativa. Esta última medición responde a que en la muestra se detectaron bajos niveles de ansiedad, a diferencia de la depresión, que se manifestaba en un mayor número. Es de notarse que dichos autores discuten el reto que constituye establecer un diagnóstico de depresión en este tipo de pacientes, pues los criterios diagnósticos para la depresión mayor incluyen síntomas asociados al cáncer, pudiéndose confundir el origen mismo del síntoma en el cáncer o en la depresión.

Diz, Garza, Olivas, Montes y Fernández (2019) ponen de manifiesto la necesidad de considerar a la familia del paciente como parte integral del tratamiento al señalar que: "La familia funge como la mayor fuente de apoyo social, personal, afectivo y económico; es por ello que el profesional de la salud mental debe abordar las posibles vicisitudes que surgen en el grupo de apoyo familiar o social del paciente. Esto significa que los conceptos que se han mencionado aquí, como optimismo, mindfulness, autotrascendencia y, en general, salutogénesis deberían considerarse en las intervenciones que se efectúan en grupos terapéuticos o de apoyo que se ofrecen a las parejas, familia o cuidadores de los pacientes, además de que los profesionales de la salud a cargo de los tratamientos y el personal médico y de enfermería requieren recibir entrenamiento para el adecuado manejo del paciente oncológico deprimido.

Flensborg-Madsen, Ventegodt y Merrick (2006) propusieron una diferenciación entre los conceptos de mentalidad y emocionalidad al suponer que hay tres entidades en el ser humano que son portadoras de consciencia: la unidad humana (el alma), el cerebro-mente (el ego y las funciones mentales) y el cuerpo-mente (el id). En ese entorno, aseveran los autores, las funciones mentales son dominadas por el cerebro, incluidas las modalidades somatosensorias, mientras que la funciones emocionales están relacionadas con el cuerpo-mente, incluyendo las relativas a la sexualidad. El concepto de psiquis humana contiene todas esas dimensiones, incluida la conciencia, relacionada con la unidad humana o alma.

Con base en estos elementos se construyó la medida Sentido de Coherencia Emocional (SOC-E, por sus siglas en inglés), que incluye cuatro emociones: enojo, alegría, pena y deseo sexual, así como tres dimensiones: intensidad, permisividad e impacto. El instrumento diseñado fue probado en una muestra de 113 individuos bajo la hipótesis de que la emocionalidad, y no la mentalidad, es el verdadero predictor de la salud física.

El estudio, realizado en Dinamarca por Flensborg-Madsen et al. (2006), probó que el SOC-E está relacionado de un modo importante con la salud física, pero no con la salud psicológica de los individuos. Mediante un refinamiento basado en los resultados obtenidos, los autores diseñaron la escala SOC-E II, considerando únicamente la intensidad y permisividad de la alegría y el deseo sexual, y dejando fuera las emociones de enojo y pena; con ello se obtuvo una mayor asociación con la salud física y psicológica. Los resultados se compararon con los de otros estudios similares, encontrándose que las emociones positivas se asocian con tasas de sobrevivencia y longevidad, mientras que los síntomas de depresión son predictores de una disminución de la sobrevivencia en pacientes con enfermedades de la arteria coronaria. Los autores concluyen lo siguiente: "En un estudio anterior que investigaba la teoría original de Antonovsky, encontramos que una operacionalización mental del SOC está altamente asociada con la salud psicológica, pero no con la salud física [...] Con base en los dos estudios, concluimos que la salud física y psicológica deben tener diferentes etiologías: la salud física tiene que ver con las emociones, mientras que la psicológica lo hace con la mentalidad" (Flensborg-Madsen et al., 2006).

Por su parte, Kröz, Büssing et al. (2009), consideran que el SOC de Antonovsky es importante por su relevancia en la prognosis de pacientes de cáncer; sin embargo, las escalas conocidas han sido validadas principalmente en pacientes 
psiquiátricos y psicosomáticos, por lo cual propusieron una escala de coherencia interna (ICS, por sus siglas en inglés) que aborda aspectos específicos para pacientes oncológicos relacionados con su fiabilidad, validez y sensibilidad a la quimioterapia. Su estudio, realizado en Alemania, y llevado a cabo en tres momentos y con diferentes muestras poblacionales, encontró que la ICS de diez reactivos demuestra tener una relevancia estadística significativa para poder considerarse como un instrumento interesante para capturar los sentimientos de coherencia interna y resistencia entre los pacientes de cáncer.

Rohani, Abedi, Omranipour y Langius-Eklöf (2015) efectuaron, en Irán, una investigación longitudinal con 162 pacientes de cáncer de mama. Los momentos del estudio fueron, a saber: T1-base o fase de prediagnosis y T2-post o seis meses después de la diagnosis. Los objetivos del estudio eran investigar los cambios en la calidad de vida relacionada con la salud (HRQoL, por sus siglas en inglés), SOC, espiritualidad y afrontamiento religioso en una muestra de mujeres con cáncer de mama. La metodología incluyó también a un grupo de control de 210 mujeres sin cáncer. Los resultados obtenidos indicaron que las mujeres con cáncer de mama calificaban peor la función física, fatiga y dificultades financieras durante la primera fase T1 que las del grupo de control. Sin embargo, a lo largo de los seis meses de estudio mejoraron su calidad de vida y funcionamiento emocional. Los autores concluyen que la recopilación de datos de HRQoL antes del diagnóstico de cáncer de mama es importante para identificar a las mujeres en riesgo de deterioro de su calidad de vida durante y después del tratamiento, subrayándose que el grado de SOC puede ser más importante como predictor de cambios en la calidad de vida que la espiritualidad y el afrontamiento religioso. Adicionalmente, sostienen que el estudio corrobora la sugerencia de Antonovsky sobre la aplicabilidad transcultural del concepto de sentido de coherencia en la predicción de cambios en la calidad de vida relacionada con la salud.

En Dinamarca, el Comité de la Ciencia y la Ética de Copenhague ha aprobado la investigación de la calidad de vida, al igual que la medicina holística. Así, Ventegodt, Omar y Merrick (2011) revisaron los estudios hechos en más de 2 mil pa- cientes con enfermedades crónicas o disfuncionales, en los que se investigaron los efectos de las intervenciones salutogénicas orientadas a mejorar la calidad de vida. Particularmente, revisaron veinte reportes acerca de dicha calidad como medicina para enfermedades como cáncer, padecimientos coronarios, dolor crónico, enfermedad mental, disfunción sexual, baja autoestima, baja capacidad de trabajo y mala calidad de vida. La estrategia de intervención más exitosa parece ser la que crea un vínculo materno, una liga de tipo infantil, inducida por una combinación de terapia de conversación y trabajo con el cuerpo. Los autores concluyen que la calidad de vida, "como medicina", es útil en el tratamiento de desórdenes físicos y otras enfermedades al ayudar a curar, o en definitiva curar, de 30 a $90 \%$ de los pacientes, típicamente en un periodo menor o igual a un año, pues mejora significativamente los problemas de salud mental, somática y sexual sin efectos secundarios.

A decir por Jonas, Beckner y Coulter (2016), "por más de 200 años la biomedicina ha abordado el tratamiento de enfermedades estudiando los procesos de la enfermedad (patogénesis), infiriendo conexiones causales y desarrollando enfoques para interferir terapéuticamente esos procesos". Esta perspectiva patogénica ha sido exitosa en las enfermedades agudas y traumáticas, pero menos con las crónicas, principalmente por su complejidad y naturaleza multifactorial; por ello, los autores señalados propusieron un modelo integrado para la evaluación de la curación (IMEH, por sus siglas en inglés).

De acuerdo con dichos autores, por investigaciones del efecto placebo y la medicina conductual se sabe que la manipulación del contexto social y cultural de la terna practicante-paciente- familia, sus estrategias de comunicación, el entorno físico y la retroalimentación de la información en el área de la salud, se puede cambiar notablemente los resultados, a menudo con un mayor alcance, que los medicamentos y los tratamientos quirúrgicos inclusive. Por ello, diseñaron el modelo siguiente. En sus palabras:

El IMEH es un enfoque que parte de la recopilación e integración de información seleccionada de la investigación sociológica, antropológica y de comportamiento, y de la biología celular 
y molecular. Lo hace desarrollando un equipo multidisciplinar y abordando la evaluación de la medicina integrativa para el cuidado del cáncer desde varias dimensiones [...] Los componentes de esa evaluación son recogidos por un equipo de investigación especializado que los alimenta en un centro integrador de datos para su análisis, modelación y síntesis [...] Adicionalmente, los datos estructurados pueden procesarse utilizando tecnología de inteligencia artificial que permita la retroalimentación, en tiempo real, de esta información al paciente y al equipo de cuidado de salud (Jonas et al., 2016).

En Alemania, de acuerdo con Weis (2003), se han establecido grupos de soporte para pacientes de cáncer en las clínicas de rehabilitación. Uno de sus objetivos generales es la promoción de los recursos individuales de los pacientes, lo cual ayuda también a las parejas y familia a aligerar el estrés asociado a la enfermedad. Durante las dos últimas décadas se han desarrollado una serie de intervenciones psicológicas que son administradas en los grupos de soporte y que utilizan métodos tales como la relajación e imaginación guiada, la educación en salud, el entrenamiento neuropsicológico, el entrenamiento conductual, la terapia con arte y ergonómica, la psicoeducación y la información didáctica.

Weis (2003) da cuenta de investigaciones que enfatizan que negar los sentimientos, las preocupaciones, el desamparo y el aislamiento social están correlacionadas con una menor salud y calidad de vida en pacientes de cáncer. Por el contrario, otros estudios empíricos demuestran que ciertas intervenciones estructuradas para grupos de pacientes mejoran su bienestar psicológico, reducen la ansiedad y la depresión, acrecientan la calidad de vida, el afrontamiento y el ajuste mental. No obstante, se estima que en Europa hay una considerable falta de unidades psicooncológicas, por lo que el apoyo está limitado a una minoría de tales pacientes.

En Polonia, Gwiazdzinski, Fedyk, Krawczyk y Szymansky (2017) llevaron a cabo un estudio cuyo objetivo era probar la hipótesis de que la práctica del yoga es benéfica para la salud física y mental. Se investigó el desarrollo que tienen el sentido de coherencia y el de agencia o control en una muestra de individuos que habían practicado hatha-yoga frente a otros (un grupo de control) que nunca lo habían hecho. Utilizando instrumentos como el experimento de la mano ajena (TAHE, por sus siglas en inglés), cuestionarios de medición del SOC y entrevistas microfenomenológicas, concluyeron que hay indicaciones de que la práctica del hatha-yoga acrecienta el sentido de control, lo cual, en el largo plazo, puede contribuir a tener una mejor salud mental. Cabe destacar que el experimento TAHE es una técnica de corte neurofenomenológico que permite evaluar el sentido de control o de agencia que tiene que ver con desórdenes psicopatológicos, tal como se puede observar en la siguiente aseveración de dichos autores: «El concepto de sentido de agencia o sus desórdenes puede ser importante para entender la esencia de unidades nosológicas como la anorexia nerviosa, el desorden de personalidad bipolar, e incluso algunos síntomas psicopatológicos que se producen en la esquizofrenia (como la "desrealización"), tal como se muestra por experimentos realizados con TAHE por Sorensen en 2005» (Gwiazdzinski et al., 2017).

\section{CONCLUSIONES}

Es de llamar la atención que mucha de la literatura relativa a la salutogénesis proviene de países europeos, no obstante que el creador del constructo procede de Estados Unidos. Muy escasas publicaciones provienen de la región hispanoamericana, por lo que la carencia de trabajos académicos sobre este tópico parece acentuarse en dicha región. Específicamente, existe aquí una gran necesidad de crear grupos de apoyo psicooncológico que difundan, promuevan y fortalezcan las buenas prácticas en torno a los fundamentos de la salutogénesis, el sentido de coherencia, el sentido de control, los recursos generalizados de resistencia, la espiritualidad concebida como el autoconocimiento del individuo, la meditación y el yoga.

Son muchas las evidencias que destacan los beneficios de la salutogénesis ya desde las fases iniciales del cáncer, por lo que se podría inferir, a reserva de investigarlo con rigor, que también puede haber una relación causal entre la práctica salutogénica y el estado de salud de quienes no 
sufren esta enfermedad u otros padecimientos, lo cual abre todo un campo de estudio en el área de la prevención de enfermedades.

Se debe resaltar también que la palabra "espiritualidad" se menciona en varias de las fuentes revisadas, aunque no se ahonda demasiado en ella. Es decir, se utiliza el concepto de bienestar físico, psicológico y espiritual, sin explicar cabalmente cómo se obtiene este último, abriéndose así otra área de investigación que permita poner a prueba las relaciones entre la espiritualidad, la salutogénesis y la preservación del bienestar integral del individuo.
Con el estado actual de la ciencia y del fenómeno oncológico, se hace necesaria la creación de protocolos, públicos o privados, para ofrecer a la población tratamientos, sobre todo psicológicos, que permitan descubrir la dimensión salutogénica y el sentido de coherencia para, entonces, promover su fortalecimiento con el fin de coadyuvar al restablecimiento de la salud de aquellas personas que padecen cáncer, y no solo la de ellas, sino también la de aquellas que sufren otro tipo de padecimientos, y aun de las que gozan de salud, para que la preserven.

\section{REFERENCIAS}

Abernethy, H. (2011). Promoting emotional wellbeing through social prescribing. En S. Cartwright y C. Cooper (Eds.): Innovations in stress and health (pp. 107-152). Basingstoke (UK ): Palgrave Macmillan.

Alemán, J. y Larriera, S. (2005). Introducción al psicoanálisis lacaniano. En J. Romero y R. Vázquez (Eds.): Psicópolis. Paradigmas actuales y alternativos en la psicología contemporánea (pp. 118-165). Barcelona: Kairós.

Alivia, M., Guadagni, P. y Roberti S., P. (2011). Towards salutogenesis in the development of personalised and preventive healthcare. EPMA Journal, 2(4), 381-384. Doi:10.1007/s13167-011-0131-9 (20/03/2019).

Antonovsky, A. (1979). Health, stress and coping. San Francisco, CA : Jossey-Bass.

Antonovsky, A. (1987). Unraveling the mystery of health. How people manage stress and stay well. San Francisco, CA: Jossey-Bass.

Berenzon, S., Lara, M., Robles, R. y Medina, M. (2013). Depresión: estado del conocimiento y la necesidad de políticas públicas y planes de acción en México. Salud Pública de México, 55(1), 74-80.

Cabrera, Y., López, J., López, E. y Arredondo, B. (2017). La psicología y la oncología: en una unidad imprescindible. Revista Finlay, 7(2), 115-127.

Campbell, J., Trapnell, P., Heine, S., Katz, I., Lavallee, L. y Lehman, D. (1996). Self-concept clarity: Measurement, personality correlates, and cultural boundaries. Journal of Personality and Social Psychology, 70(1), 141-156.

Dias de Andrade, F. (2013). The impact of self-concept clarity on information processing of college students. Millersville, PA: Millersville University.

Diz, R., Garza, A., Olivas, E., Montes, J. y Fernández, G. (2019). Cáncer y depresión: una revisión. Psicología y Salud, 29(1), $115-124$

Eriksson, M. (2007). Unravelling the mystery of salutogenesis. The evidence base of the salutogenic research as measured by Antonovsky's Sense of Coherence Scale. Helsinki: Folkhalsan Research Centre.

Flensborg-Madsen, T., Ventegodt, S. y Merrick, J. (2006). Sense of coherence and physical health. The emotional sense of coherence (SOC-E) was found to be the best-known predictor of physical health. TSW Holistic Health \& Medicine, 1, $183-193$.

Friedman, L., Kalidas, M., Elledge, R., Chang, J., Romero, C., Husain, I., Dulay, M. y Liscum, M. (2005). Optimism, social support and psychosocial functioning among women with breast cancer. Psycho-Oncology, 15(7), 595-603. Doi: 10.1002/ pon.992.

Gerasimcik-Pulko, V., Pileckaite-Markoviene, M., Bulotiene, G. y Ostapenko, V. (2009). Relationship between sense of coherence and quality of life in early stage breast cancer patients. Acta Medica Lituanica, 16(3-4), 129-144.

Guerrero, J., Prepo, A. y Loyo, J. (2016). Autotrascendencia, ansiedad y depresión en pacientes con cáncer en tratamiento. Revista Habanera de Ciencias Médicas, 15(2), 297-309. Recuperado de https://doi.org/10.1016/j.rccan.2015.04.005 (06/04/2019).

Gustavsson-Lilius, M., Julkunen, J., Keskivaara, P., Lipsanen, J. y Hietanen, P. (2010). Predictors of distress in cancer patients and their partners - The role of optimism in the sense of coherence construct. Psychology \& Health, 27(2), 178-195. Doi: 10.1080/08870446.2010.484064.

Gwiazdzinski, P., Fedyk, O., Krawczyk, M. y Szymansky, M. (2017). Practicing hatha-yoga, sense of coherence, and sense of agency. Neurophenomenological approach. Psychiatria Danubina, 29(Suppl. 3), 530-535. 
Hillman, J. (1975). Re-visioning psychology. New York: Harper and Row.

Jonas, W., Beckner, W. y Coulter, I. (2016). Proposal for an integrated evaluation model for the study of whole systems health care in cancer. Integrative Cancer Therapies, 5(4), 315-319. Doi: 10.1177/1534735406295565.

Jung, C.G. (1997). Jung on active imagination. (Ed. J. Chodorow). Princeton, NJ: Princeton University Press.

Kröz, M., Büssing, A., Von Laue, H., Reif, M., Feder, G., Schad, F., Girke, M. y Matthes, H. (2009). Reliability and validity of a new scale on internal coherence (ICS) of cancer patients. Health and Quality of Life Outcomes, 7(59). Doi: 10.1186/14777525-7-59.

Lindström, B. y Eriksson M. (2006). Contextualizing salutogenesis and Antonovsky in public health development. Health Promotion International, 21(3), 238-244.

Mota, C., Aldana, E., Bohórquez, L., Martínez, S. y Peralta, J. (2018). Ansiedad y calidad de vida en mujeres con cáncer de mama: una revisión teórica. Psicología y Salud, 28(2), 155-165.

Organización Mundial de la Salud (1946). Constitución de la Organización Mundial de la Salud. Ginebra: OMS.

Pousa, V., Miguelez, A., Hernández, M., González, M. y Gaviria, M. (2015). Depresión y cáncer: una revisión orientada a la práctica clínica. Revista Colombiana de Cancerología, 19(3), 166-172.

Rivera, F., Ramos, P., Moreno, C. y Hernán, M. (2011). Análisis del modelo salutogénico en España: aplicación en salud pública e implicaciones para el modelo de activos en salud. Revista Española de Salud Pública, 85(2), 129-139.

Rodríguez, A., Casas, D., Contreras, G. y Ruano, L. (2015). De la enfermología a la salutogénesis: conceptos teóricos en la búsqueda de la salud como derecho universal. Revista Médica de la Universidad de Costa Rica, 9(2, Art. 1).

Rohani, C., Abedi, H., Omranipour, R. y Langius-Eklöf, A. (2015). Health-related quality of life and the predictive role of sense of coherence, spirituality and religious coping in a sample of Iranian women with breast cancer: a prospective study with comparative design. Health and Quality of Life Outcomes, 13(40). Doi: 10.1186/s12955-015-0229-1.

Ruano, L. y Mercé, E. (2014). Estado actual de la salutogénesis en España. Quince años de investigación. Enfermería Global, 13(2), 384-405. Doi: 10.6018/eglobal.13.2.165721.

Saravia, J., Iberico, C. y Yearwood, K. (2014). Validation of sense of coherence (SOC) 13-item scale in a Peruvian sample. Journal of Behavior, Health \& Social Issues, 6(2).

Ventegodt, S., Omar, H. y Merrick, J. (2011). Quality of life as medicine: interventions that induce salutogenesis. A review of the literature. Social Indicators Research, 100(3), 415-430.

Volanen, S., Lahelma, E., Silventoinen, K. y Suominen, S. (2004). Factors contributing to sense of coherence among men and women. European Journal of Public Health, 14(3), 322-330. Doi:10.1093/eurpub/14.3.322.

Wainwright, N., Surtees, P., Welch, A., Luben, R., Khaw, K. y Bingham, S. (2007). Healthy lifestyle choices: could sense of coherence aid health promotion? Journal Epidemiology and Community Health, 61(10), 871-876.

Weis, J. (2003). Support groups for cancer patients. Supportive Care Cancer, 11(12), 763-768. Doi: 10.1007/s00520-003-0536-7. 